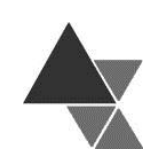

\title{
Condições higiênico-sanitárias de estabelecimentos de alimentação de um parque multitemático no Vale do Itajaí, Santa Catarina, Brasil
}

\author{
Elisabeth Barth Almeida ${ }^{1}$, Rosana Henn ${ }^{1}$, Daniela Carolina Feder ${ }^{2}$ e Sandy Aparecida \\ Bartholo ${ }^{2}$
}

As doenças transmitidas por alimentos podem estar relacionadas com as inadequações no controle higiênicosanitário. A partir dessa consideração, o objetivo deste estudo foi avaliar o nível de conformidade do controle higiênico-sanitário dos pontos terceirizados que comercializam alimentos em um parque multitemático do Vale do Itajaí (SC), antes e após auditoria, de acordo com a Resolução da Diretoria Colegiada no 216/2004 da Agência Nacional de Vigilância Sanitária. Foram avaliados 41 estabelecimentos do setor de alimentação, por meio de um check-list higiênico-sanitário próprio do parque, adaptado para cada categoria de estabelecimento: restaurantes, lanchonetes e confeitaria, sorveterias, cafeterias, bombonieres (industrializados) e pipoqueira, sorveteria e cafeteria. Após análise dos dados, os estabelecimentos foram classificados pela pontuação alcançada. Estabeleceu-se um prazo para melhoria das não conformidades, sendo realizada uma segunda auditoria após o prazo determinado. A maioria dos estabelecimentos não atingiu o percentual preconizado pela empresa na primeira auditoria e, após o retorno, prevaleceram as classificações nas categorias "bom" e "muito bom". Foi possível verificar a importância da auditoria, uma vez que os percentuais de conformidade aumentaram entre elas.

Palavras-chave: Boas Práticas de Manipulação; Segurança dos Alimentos e Nutricional; Controle de Qualidade.

\section{Hygienic-sanitary conditions of food establishments in a multi-thematic park in the Itajaí Valley, Santa Catarina, Brazil}

Foodborne illness may be related to the inadequacies in the hygienic-sanitary control. The aim of this study was to evaluate the level of compliance of hygienic-sanitary control of outsourced foods marketed in a multi-thematic Park at Vale do Itajaí (SC), before and after audit, according to the Resolution 216/2004 of the National Health Surveillance Agency. For that, 41 establishments were evaluated, through a hygienic and sanitary check-list, adapted for each category of establishment, including restaurants, snack and confectionery, ice cream parlors, coffee shops, bombonieres (industrialized) and popper, ice cream parlor and coffee shop. After analyzing the data, the establishments were scored, and a deadline was defined for improvement of nonconformity, followed by a second audit. Most establishments did not reach the percentage recommended by the company in the first audit, and the rankings as "good" and "very good" prevailed within the categories in the second audit. The audit has proven to be an effective tool, since an increase in compliance percentage was observed between auditing.

Keywords: Good Manipulation Practices; Food and Nutrition Security; Quality Control.

\footnotetext{
${ }^{1}$ Professora do Curso de Nutrição. Universidade do Vale do Itajaí. Endereço para correspondência: Rua Uruguai no 458, Centro, Itajaí, Santa Catarina, CEP: 88302-202. Tel.: +55 (47)3341-7897/9915-9575. E-mail: bethbarth@univali.br 


\section{INTRODUÇÃO}

A qualidade na produção de alimentos é primordial para a obtenção de produtos seguros para os consumidores. Isso implica manter o controle higiênico, que se refere a toda e qualquer ação que visa melhorar as Boas Práticas de Manipulação (BPM) na preparação dos alimentos. Já o controle sanitário é definido como qualquer tipo de ação que visa melhorar os processos e atribuir segurança nas preparações ${ }^{[1]}$. As BPM são procedimentos para atingir um padrão de qualidade de um produto ou serviço na área de alimentação ${ }^{[2,3]}$. É direito de todos os indivíduos ter expectativa de que os alimentos que consomem sejam seguros e adequados ao consumo. A segurança dos alimentos é um desafio à saúde pública, uma vez que visa oferecer alimentos inócuos aos cidadãos e manter a integridade da saúde do consumidor ${ }^{[3]}$.

A manipulação inadequada é apontada como a principal causa de contaminação dos alimentos em todo o mundo ${ }^{[4]}$. Inadequações do controle higiênicosanitário podem causar Doenças Transmitidas por Alimentos (DTA), que ocorrem devido à contaminação por microrganismos como bactérias, vírus, parasitas e toxinas, por produtos químicos, agrotóxicos e metais pesados ou outros contaminantes ${ }^{[5]}$.

Com o intuito de prevenir e evitar a ocorrência desses problemas, foram instituídos padrões e diretrizes nos âmbitos federal, estadual e municipal para indústrias e serviços de alimentação. A necessidade de padronizar as BPM nas empresas processadoras de alimento se deve ao fato de que o governo necessita zelar pela saúde da população, uma vez que o consumidor não possui condições para identificar os atributos higiênico-sanitários de cada estabelecimento $^{[6]}$.

A auditoria tem como objetivo zelar pela segurança dos alimentos e qualidade. Quando devidamente realizada, gera benefícios para a gestão do estabelecimento, tanto no setor operacional, como no estratégico. A auditoria não garante totalmente a qualidade dos produtos e serviços, no entanto, é um método bastante utilizado para controlá-los e melhorá-los ${ }^{[7]}$.
Nesse contexto, a Agência Nacional de Vigilância Sanitária (ANVISA), por meio da Resolução da Diretoria Colegiada - RDC no 216, de setembro de 2004, estabelece que os serviços de alimentação devem seguir procedimentos de boas práticas a fim de garantir as condições higiênicosanitárias desde a avaliação criteriosa dos fornecedores, passando por cuidados durante o recebimento, armazenamento, higienização do ambiente, higiene pessoal, produção e exposição do alimento ${ }^{[2]}$.

Para que sejam mantidas condições satisfatórias durante todas as etapas do processo de produção e manipulação, recomenda-se o acompanhamento das atividades por um responsável devidamente capacitado. Já a responsabilidade técnica fica a cargo de um profissional nutricionista, o qual deve elaborar o Manual de Boas Práticas (MBP) e os Procedimentos Operacionais Padronizados (POP's), capacitar continuamente os manipuladores de alimentos e supervisionar diariamente a produção. Somente com essas ações é possível alcançar melhores padrões de qualidade ${ }^{[8]}$.

A alimentação que atende os padrões higiênicos e satisfatórios é uma das condições essenciais para a promoção e a manutenção da saúde, sendo que a deficiência nesse controle é um dos fatores responsáveis pela ocorrência de surtos de doenças transmitidas por alimentos ${ }^{[9]}$.

O objetivo do presente estudo foi avaliar o nível de conformidade do controle higiênico-sanitário dos pontos terceirizados que comercializam alimentos em um parque multitemático do Vale do Itajaí (SC), antes e após auditoria.

\section{METODOLOGIA}

Estudo realizado no maior parque multitemático da América Latina, foi submetido à aprovação do Comitê de Ética da Universidade do Vale do Itajaí e aprovado pelo parecer no ${ }^{-}$819.887. Trata-se de um estudo transversal, no qual apresentavam 44 estabelecimentos sendo que destes, 41 aceitaram participar do estudo e posteriormente foram divididos em cinco categorias: restaurantes / lanchonetes, bombonieres (industrializados), 
sorveterias, cafeterias e sorveterias/cafeterias. A classificação foi determinada pelo setor de varejo e patrocinadores do parque. Todos os proprietários dos estabelecimentos que concordaram em participar da pesquisa assinaram o Termo de Consentimento Livre e Esclarecido (TCLE).

A coleta de dados ocorreu no período de outubro de 2014 a janeiro de 2015, por meio da aplicação de uma lista de verificação higiênicosanitária (check-list) específica para cada uma das cinco categorias. Todos os critérios de avaliação tiveram como base o check-list higiênico sanitário já utilizado no parque, adaptado da RDC no 216 da ANVISA ${ }^{[2]}$. A lista de verificação higiênico-sanitária foi padronizada com perguntas fechadas e cada item foi avaliado por meio de observação direta de acordo com as categorias: Conforme (C), Não Conforme (NC) e Não se Aplica (NA). Para realizar a pontuação, o check-list foi dividido em cinco itens: 1) Documentação: manual de boas práticas, alvará sanitário e de localização; 2) Instalações Físicas/Ambiente: área externa, pisos, forros e tetos, paredes, divisórias e pilares, portas, janelas, ralos e canaletas, iluminação, ventilação, higienização das instalações, lavatórios, ambiente de trabalho, instalações físicas, água, lixo e esgoto, caixa; 3) Equipamentos, Utensílios e Móveis; 4) Manipuladores de Alimentos e Atendentes: uniforme, comportamento, atestado de saúde, treinamento, higienização de mãos, Equipamentos de Proteção Individual (EPI), visitantes; 5) Área de Produção: recebimento, armazenamento, procedimentos operacionais. Esses dados forneceram informações que possibilitaram a classificação geral dos estabelecimentos. Os check-lists foram aplicados nos pontos avaliados pelas pesquisadoras com acompanhamento da nutricionista do departamento de varejo patrocinador do parque.

Após análise dos dados, os estabelecimentos foram classificados pela pontuação padronizada pelo parque: $100 \%$ excelente, 91 a $99 \%$ muito bom, 81 a $90 \%$ bom, 61 a $80 \%$ regular e até $60 \%$ deficiente. Quando necessário, foram notificadas ações corretivas para as não conformidades encontradas, com prazo determinado de 15 a 20 dias para execução de melhorias.
As não conformidades dos estabelecimentos foram notificadas pela nutricionista supervisora de qualidade, com uso de formulário específico de notificações de infração contratual, conforme consta no regulamento interno da empresa e no contrato entre a locadora e o locatário. Após o prazo estabelecido, foi realizado um retorno ao local para verificar se as "não conformidades" foram corrigidas. Em seguida foram elaborados relatórios para os proprietários, contendo a sua classificação que, segundo o Departamento de Varejo e Patrocínio, deve estar entre $91 \%$ e $100 \%$, ou seja, nas categorias "excelente" e "muito bom", podendo ocorrer notificações e multas caso não haja adequação.

Os dados coletados na pesquisa foram tabulados com auxílio dos programas Microsoft Excel ${ }^{\circledR}$ e Microsoft Word ${ }^{\circledR} 2010$.

\section{RESULTADOS E DISCUSSÃO}

Em relação aos 41 estabelecimentos analisados, $43,90 \% \quad(n=18)$ eram da categoria restaurantes/lanchonetes, $\quad 29,26 \% \quad(n=12)$ bombonieres (industrializados), 12,20\% $\quad(n=5)$ sorveterias, 9,76\% $(n=4)$ cafeterias e 4,88\% $(n=2)$ sorveterias e cafeterias. Foi possível verificar a classificação dos estabelecimentos entre a auditoria e o retorno.

Com relação à documentação, item 1 do check-list, avaliou-se, a presença de três itens: manual de boas práticas, alvará sanitário e alvará de localização (Figura 1).

Figura 1. Distribuição percentual dos estabelecimentos, divididos por categorias, em relação à documentação, 2015

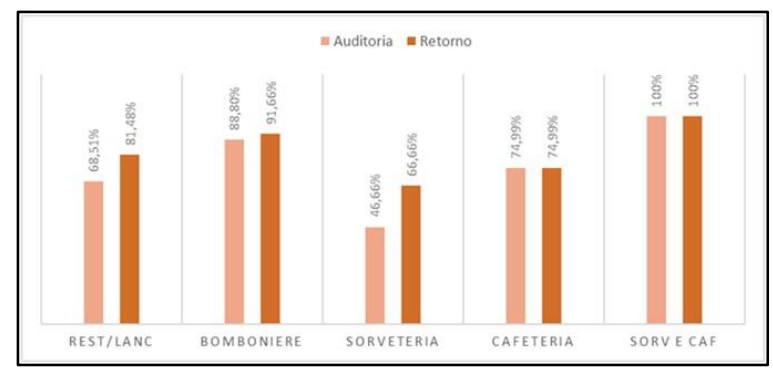

De acordo com a Figura 1, a categoria "sorveteria e cafeteria" apresentou o melhor 
percentual, com 100\% (excelente) na auditoria e no retorno. Já os restaurantes/lanchonetes e as sorveterias obtiveram o menor percentual de adequação na auditoria, mas, embora tenha sido verificada melhora no retorno, esses estabelecimentos não conseguiram se classificar dentro padrão estabelecido. $\mathrm{Na}$ cafeteria não houve diferença entre a auditoria e o retorno, mantendo-se abaixo do preconizado. As bombonieres não apresentaram a classificação desejada na auditoria, mas no retorno alcançaram o percentual preconizado pela empresa. Dentre os itens avaliados na documentação, o que se mostrou mais deficiente foi o alvará sanitário. Isso ocorreu devido ao atraso do órgão municipal de vigilância sanitária na entrega dos documentos. Todos os proprietários relataram terem pago o alvará, porém, a maioria não o recebeu. Segundo a ANVISA, o alvará sanitário e de funcionamento é um documento expedido por órgão municipal competente, que autoriza o funcionamento de estabelecimentos que realizam atividades sob regime de vigilância sanitária e que devem possuir licença sanitária atualizada de acordo com a legislação sanitária local, fixada em local visível ao público ${ }^{[9]}$.

Em relação ao $\mathrm{MBP}$, todos os estabelecimentos apresentaram 100\% de aprovação. Já Oliveira, Santana e Silva ${ }^{[10]}$ avaliaram as condições higiênico-sanitárias de restaurantes localizados na Barra do Garças (MT), por meio de um check-list, e observaram, no item documentação, a inexistência do MBP.

Em relação às instalações físicas/ambiente, foram avaliados 77 itens (Figura 2).

Figura 2. Distribuição percentual dos estabelecimentos, divididos por categorias, em relação às instalações físicas/ambiente, 2015

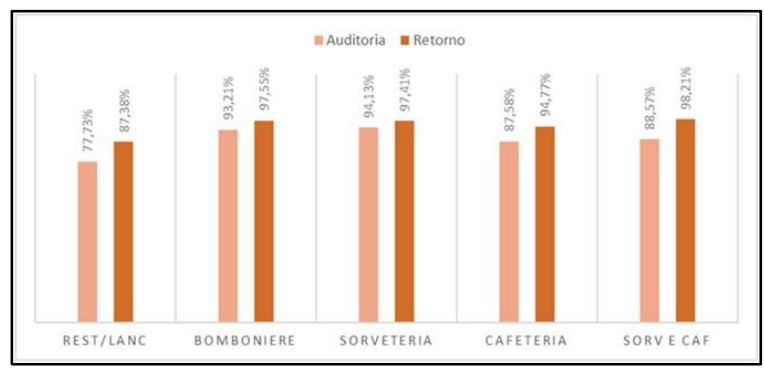

Observa-se, na Figura 2, que o maior índice de inadequação foi na categoria restaurantes/lanchonetes, havendo melhora no retorno, sem que os estabelecimentos tenham, contudo, conseguido alcançar o percentual desejado. As categorias bombonieres e sorveteria atingiram o percentual preconizado nas duas visitas. $\mathrm{Na}$ primeira auditoria, cafeterias e sorveterias/cafeterias não se classificaram de acordo com o percentual desejado e, no retorno, as duas categorias conseguiram alcançar a classificação preconizada.

Uma das justificativas para as inadequações das instalações físicas/ambiente consiste no fato de os estabelecimentos terem se adaptado a um espaço físico já existente, não tendo este sido construído para atender as necessidades preconizadas pela legislação para uma Unidade Produtora de Refeição (UPR). Sobre esse aspecto, pesquisa realizada por Melo et al. [11] revelou que, quanto à estrutura física, $70 \%$ dos restaurantes populares estudados foram adaptados em locais já construídos.

A maioria das inadequações encontradas, em todas as categorias foi em relação a pisos, paredes, ralos, caneletas e equipamentos de ventilação em mau estado de conservação e luminárias sujas e sem proteção. Ressalta-se que as adequações desses itens devem ser realizadas pelos locatários dos estabelecimentos, de acordo com a legislação vigente.

Os pisos, paredes e tetos devem possuir revestimento liso, impermeável e lavável. Devem ser mantidos íntegros, conservados, livres de rachaduras, trincas, goteiras, vazamentos, infiltrações, bolores e descascamentos, de modo a não transmitirem contaminantes aos alimentos [2]. Estes fatores dificultam a limpeza das instalações e dos equipamentos, podendo acarretar em contaminações cruzadas, e as luminárias sem proteção podem causar acidentes, trazendo danos aos manipuladores e na produção de alimentos.

Estudo conduzido por Costa et al. ${ }^{[12]} \mathrm{em}$ Goiânia (GO), avaliou 27 restaurantes do tipo selfservice. A maioria mostrou condições precárias de piso, paredes e tetos com rachaduras, não apresentando cores claras o que dificulta a higienização, portas sem fechamento automático, janela e ralos sem proteção. 
Capelesso e Hautrive ${ }^{[13]}$, por meio de investigação realizada em Chapecó (SC), avaliaram 10 restaurantes comerciais. Em relação às instalações físicas, observaram, em $80 \%$ dos restaurantes, que os tetos, pisos e paredes não eram mantidos íntegros, apresentaram-se mal conservados, com rachaduras, trincas e descascamento.

Os equipamentos, móveis e utensílios foram avaliados a partir de 16 itens (Figura 3).

Figura 3. Distribuição percentual dos estabelecimentos, divididos por categorias, em relação aos equipamentos, 2015

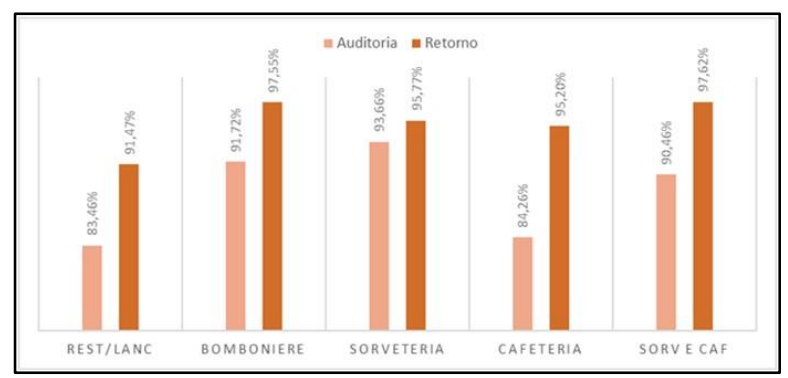

Os resultados obtidos, conforme a Figura 3, mostram que na primeira auditoria, os restaurantes/lanchonetes, as cafeterias e sorveterias/cafeterias apresentaram condições inferiores ao que é preconizado. Todavia, no retorno conseguiram atingir o percentual desejado. As categorias bombonieres e sorveterias atingiram o percentual necessário nas duas auditorias.

Observou-se maior deficiência em relação ao estado de conservação e funcionamento: termômetros sem calibração, que podem levar ao erro na mensuração das temperaturas, aumentando o risco da proliferação de microorganismos nos alimentos, equipamentos, móveis e utensílios sujos, podendo acarretar em contaminação cruzada e falta de registro de manutenção dos equipamentos. Equipamentos mal conservados e com funcionamento deficiente, podem causar despesas extras aos estabelecimentos devido a um gasto maior de energia; podendo também prejudicar a área de produção, já que por apresentarem mau funcionamento não conseguirão suprir a demanda do local, podendo também influenciar a segurança dos alimentos. É importante ressaltar que os equipamentos, móveis e utensílios devem ser mantidos em adequado estado de conservação e ser resistentes à corrosão e a repetidas operações de limpeza e desinfecção [2]. Os equipamentos precisam funcionar adequadamente, possuir termômetros bem regulados e a manutenção deve ser constantemente realizada ${ }^{[14]}$.

Estudo realizado por Machado, Gonçalves e Pereira ${ }^{[15]}$ avaliou 47 panificadoras na cidade de Bauru (SP). No item referente aos equipamentos e utensílios, as maiores inconformidades foram associadas à limpeza/desinfecção $(44,7 \%)$ e ao armazenamento $(51,1 \%)$.

Ferreira et al. [16] verificaram, em equipamentos, móveis e utensílios, um percentual de adequação de $71,4 \%$ a $90,5 \%$. Os problemas mais frequentes foram relacionados aos equipamentos de conservação dos alimentos, que não apresentavam medidor de temperatura ou não estavam em adequado estado de funcionamento, assim como a inexistência de registros de manutenção preventiva dos equipamentos.

Em relação aos manipuladores foram avaliados 33 itens (Figura 4).

Figura 4. Distribuição percentual dos estabelecimentos, divididos por categorias, em relação aos manipuladores, 2015

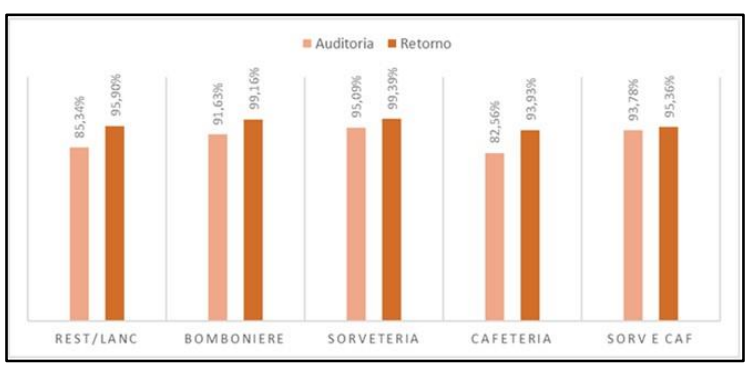

Foi possível verificar, conforme a Figura 4, que os restaurantes/lanchonetes e a cafeterias não obtiveram o percentual de adequação necessário na primeira auditoria, porém, no retorno, conseguiram se adequar ao que é preconizado. Quanto às categorias bombonieres, sorveterias e sorveterias/cafeterias, estas apresentaram, nas duas auditorias, percentuais de adequação concordante com o preconizado.

Em relação aos manipuladores, as inadequações foram carteiras de saúde e exames vencidos. Cabe acentuar que a carteira de saúde e os 
exames, segundo regulamento interno da empresa, devem ser renovados anualmente, sendo eles: Venereal Disease Research Laboratory (VDRL - teste para identificação de sífilis), fezes coprocultura e parasitológico, parcial de urina e hemograma completo. Ressalta-se que os documentos que atestem a saúde do manipulador devem permanecer à disposição da autoridade sanitária ${ }^{[14]}$. A principal finalidade dos exames e da carteira de saúde é monitorar a saúde dos trabalhadores. Desse modo, dispõe-se de recurso valioso para possível realização de diagnósticos precoces e rápidas intervenções, no caso de identificação de problemas, para que esses não causem prejuízos à saúde dos consumidores.

Com resultados semelhantes aos verificados, Vargas e Ueno ${ }^{[17]}$ avaliaram as condições de higiene na manipulação de lanches no comércio de alimentos no município de Taubaté (SP) e constataram que $74,6 \%$ dos manipuladores de alimentos não realizavam exames médicos periódicos para trabalhar nessa atividade. Outra inadequação diz respeito aos pertences pessoais guardados em local inadequado. Isso ocorreu porque a estrutura física do estabelecimento não era adequada, ou seja, não existe um espaço próprio para que os manipuladores guardem seus pertences. A RDC $\mathrm{n}^{\circ}-216$ determina que as roupas e os objetos pessoais devem ser guardados em local específico e reservado para esse fim $^{[2]}$. A área de produção contou com a avaliação de 42 itens (Figura 5).

Figura 5. Distribuição percentual dos estabelecimentos, divididos por categorias, em relação à área de produção, 2015

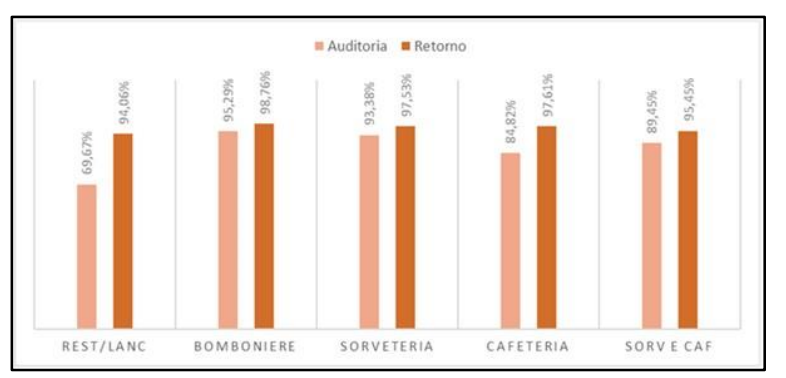

Verificou-se, como mostrado na Figura 5, que o maior índice de inadequação foi encontrado na categoria de restaurantes/lanchonetes, com um percentual muito abaixo do preconizado na auditoria. Contudo, uma grande melhora foi registrada no retorno, com alcance do percentual desejado. Nas categorias cafeteria e sorveteria/cafeteria, constatou-se que as mesmas não atingiram o percentual necessário durante a auditoria, sendo que, no retorno, ambas conseguiram atingir o percentual estabelecido. Já as categorias sorveterias e bombonieres apresentaram, tanto na auditoria quanto no retorno, a classificação preconizada.

As maiores deficiências foram encontradas na categoria restaurantes/lanchonetes e recaíram sobre os seguintes itens: ausência de preenchimentos das planilhas de recebimento, temperatura de cocção, temperatura dos equipamentos de refrigeração e distribuição, higienização de hortifrútis. Salienta-se que devem ser registrados, em planilhas, os dados referentes a recebimento, temperatura de cocção, temperatura dos equipamentos de refrigeração e distribuição e higienização de hortifrutis para controle e garantia de que os processos estão sendo realizados corretamente ${ }^{[13]}$. Segundo relato dos proprietários dos estabelecimentos, o baixo índice de preenchimento das planilhas se dá por motivos de falta de treinamento e capacitação, em virtude da grande rotatividade de funcionários.

Para Tondo e Bartz ${ }^{[18]}$, a dificuldade maior é fazer com que o manipulador entenda a necessidade e a função dos registros, pois seu foco está relacionado com a preparação dos alimentos. É necessário lembrar o manipulador que, em caso de surto ou reclamações, o registro pode ajudar na defesa.

Um estudo de Deschamps et al. ${ }^{[19]}$ avaliou unidades de alimentação e nutrição em Blumenau (SC) e concluiu que as inadequações mais frequentes foram a ausência de mensuração do binômio tempo/temperatura.

Outra inadequação encontrada nos estabelecimentos do parque foi a presença de produtos alimentícios sem etiqueta completa após abertura. A ausência de etiquetas dificulta o controle de qualidade do alimento após aberto, podendo acarretar em prejuízos a segurança dos alimentos. Para estar em conformidade com as diretrizes sanitárias, os alimentos devem possuir etiqueta com identificação, após a abertura, com nome do produto, data da abertura e data de vencimentos ${ }^{[14]}$. 
Medeiros et al. ${ }^{[20]}$ avaliaram as condições de armazenamento dos alimentos e apontaram, como principais alterações, alimentos armazenados sem invólucro, embalagens avariadas e uso de materiais inadequados para a embalagem.

Verificou-se também que os óleos e gorduras não eram trocados frequentemente, podendo afetar a qualidade nutricional e sensorial dos alimentos. As normas sanitárias preconizam que os óleos e gorduras utilizadas devem ser substituídos imediatamente sempre que houver alteração evidente das características físico-químicas ou sensoriais, tais como aroma e sabor, e formação intensa de espuma e fumaça ${ }^{[14]}$.

Bueno e Gatti ${ }^{[21]}$ avaliaram as principais atividades realizadas em serviços à la carte e self-service e constataram que o óleo utilizado nas frituras por imersão era trocado semanalmente ou de acordo com o movimento no local.

A inadequação no armazenamento das sobras limpas foi outra não-conformidade encontrada. Acentua-se que a utilização de sobras limpas pode ser realizada desde que exista um controle de tempo e temperatura em todas as etapas do preparo ${ }^{[14]}$.

Em sua investigação, Vargas e Ueno [17] observaram que as matérias-primas estavam armazenadas inadequadamente, em caixas de isopor, e mantidas em temperaturas diferentes das exigidas por critério de segurança.

Nos estabelecimentos do parque em Penha (SC) foi possível observar inadequação no processo de resfriamento dos alimentos, com a permanência dos mesmos em temperaturas que favorecem a multiplicação microbiana. A adequação do processo deve ser observada visando a minimização do risco de contaminação cruzada ${ }^{[2]}$. A deficiência nesses itens pode ser decorrente, em parte, pelo fato da coleta de dados ter sido realizada em período de alta temporada turística na região. É comum nessa época o atendimento a um número de visitantes significativamente maior do que em outros meses, o que supostamente reduz o tempo para a realização adequada das atividades, comprometendo o cumprimento de "boas práticas" nesses estabelecimentos. Cabe também registrar que a alta rotatividade de funcionários, associada à elevação da demanda, impossibilita o desenvolvimento de programas de capacitação e treinamento dos colaboradores.

\section{CONCLUSÃO}

A análise dos resultados permitiu constatar que a categoria restaurantes/lanchonetes foi a que apresentou o maior índice de deficiência em vários itens analisados pelo check-list. Entre as não conformidades observadas, destaca-se a falta do Alvará Sanitário, em virtude de atraso da entrega do documento pelo órgão municipal responsável por sua liberação. Esse resultado demonstra a falta de comprometimento do município em atender aos proprietários dos estabelecimentos que estão com as solicitações e os pagamentos dos Alvarás em dia; em contrapartida, o parque não deveria autorizar $o$ funcionamento dos mesmos sem a presença do Alvará. Ainda em relação às não conformidades verificadas, os espaços físicos apresentaram limitações, pois a maioria dos estabelecimentos foi adaptada a um local já existente e não construído especialmente para abrigar suas atividades. A alta rotatividade de funcionários também foi observada, comprometendo a dinâmica de capacitação de colaboradores.

Foi possível verificar a importância da auditoria, uma vez que os percentuais de conformidade aumentaram entre as avaliações. Salienta-se que na primeira auditoria, a maioria dos estabelecimentos não alcançou o percentual preconizado pela empresa e, após o retorno, a maioria foi classificada nas categorias "bom" e "muito bom", seguido da categoria "excelente".

A nutricionista do parque é responsável por realizar auditorias internas em todos os estabelecimentos. Essas auditorias acontecem duas vezes por ano. Além da nutricionista do parque, todos os estabelecimentos têm nutricionista consultora em sua equipe. $\mathrm{O}$ acompanhamento desse profissional é importante nas etapas dos processos visando a eliminação das inadequações encontradas e de capacitação de funcionários quanto à segurança dos alimentos, garantindo, assim, a qualidade do produto final e prevenindo riscos de contaminações por meio dos alimentos. Considerando que o parque exige nível 
de excelência no atendimento ao visitante, ressalta-se a importância de garantir a segurança dos alimentos, por meio do cumprimento de $100 \%$ das exigências avaliadas pela lista de verificação higiênico-sanitária.

\section{REFERÊNCIAS}

[1] Silva Junior EA. Manual de Controle Higiênico-Sanitário em Serviços de Alimentação. 7ed. São Paulo: Livraria Varela; 2014.

[2] Brasil. Ministério da Saúde. Agência Nacional de Vigilância Sanitária - RDC no 216, de 15 de setembro de 2004. Dispõe sobre o Regulamento Técnico de Boas Práticas para Serviços de Alimentação. Diário Oficial da União. 16 set 2004.

[3] Pilla CS. Perfil das denúncias recebidas pelo programa de alimentos da Vigilância Sanitária de Viamão/RS [monografia] [internet]. Porto Alegre: Universidade Federal do Rio Grande do Sul; 2009. [acesso em 19 mai 2014]. Disponível em: http://www.lume.ufrgs.br/bitstream/handle/10183/22912/00 0734571.pdf

[4] Soares LS. Segurança dos alimentos: avaliação do nível de conhecimento, atitudes e práticas dos manipuladores de alimentos na rede municipal de ensino de Camaçari - BA [dissertação]. Salvador: Universidade Federal da Bahia; 2011.

[5] Wingert C. Avaliação das condições higiênicos sanitárias dos serviços de alimentação de um shopping center do município de Porto Alegre [dissertação]. Porto Alegre: Universidade Federal do Rio Grande do Sul; 2012.

[6] Kaziliûnas A. Problems of auditing using quality management systems for sustainable development of organizations. Technol. Econ. Dev. Eco. 2008;4(1):64-75.

[7] Carvalho ACMS, Barbosa NP. Condições HigiênicoSanitárias de Panificadoras de uma Rede de Supermercados da Cidade de Goiânia. Rev. Nutr. em Pauta. 2013;21(120):21-25.

[8] Colombo M, Oliveira KMP, Silva LD. Conhecimento das merendeiras de Santa Fé - PR, sobre higiene e boas práticas de fabricação na produção de alimento. Rev. Hig. Alimentar. 2009;23(170/171):39-46.

[9] Brasil. Ministério da Saúde. Agência Nacional de Vigilância Sanitária - RDC nํ 11, de 16 de fevereiro de 2012. Diário Oficial da União. 14 fev 2012. Seção 1. p.23-24.

[10] Oliveira KAM, Santana ECM, Silva LR. Avaliação das condições higiênico sanitárias e do conhecimento das Boas Práticas em restaurantes self-service do município de Barra do Garças, MT. Rev. Hig. Alimentar. 2011;25(194/195):46-49.
[11] Mello AG, Sales GLP, Jaeger LM; Colares LGT. Estrutura físico-funcional de restaurantes populares do estado do Rio de Janeiro: influência sobre as condições higiênico sanitárias. Rev. Demetra. 2013;8(2):91-101.

[12] Costa CF, Oliveira FC, Ribeiro, APM, Jaime RP, Campos RC, Nojimoto ITI. Política de segurança alimentar: avaliação da utilização das boas práticas de confecção através de check-list em restaurantes de Goiânia. J. Health Sci. Inst. 2010;28(4):334336.

[13] Capelesso S, Hautrive TP. Condições higiênico-sanitárias de restaurantes comerciais de Chapecó, SC. Rev. Hig. Alimentar. 2014;28(234/235):88-92.

[14] Associação Brasileira das Empresas de Refeições Coletivas - ABERC. Manual ABERC de Práticas de Elaboração e Serviço de Refeições para Coletividades. 2013;(10):255.

[15] Machado NL, Gonçalves LP, Pereira RACB. Condições higiênico-sanitárias de panificadoras do município de Bauru, SP. Rev. Hig. Alimentar. 2011;25(202/203):28-35.

[16] Ferreira MA, São João JFB, Tomazini HSD, Milagres RCM, Pinheiro Santana HM. Avaliação da adequação às boas práticas em unidades de alimentação e nutrição. Rev. Inst. Adolfo Lutz. 2011;70(2):230-235.

[17] Vargas D, Ueno M. Higiene na manipulação de lanches no comércio ambulante de alimentos. Rev. Hig. Alimentar. 2014;28(236/237):38-43.

[18] Tondo E, Bartz B. Microbiologia e sistema de gestão de segurança de alimentos. Porto Alegre: Sulina; 2011.

[19] Deschamps C, Freygang J, Bramorski A, Tommasi D, Garcia GF. Avaliação higiênico-sanitária de cozinhas industriais instaladas no município de Blumenau, SC. Rev. Hig. Alimentar. 2003;17(112):12-15.

[20] Medeiros L, Dall'agnol LP, Botton SA, Smaniotto H, Potter R, Campos MMA, et al. Qualidade higiênico-sanitária dos restaurantes cadastrados na Vigilância Sanitária de Santa Maria, RS, Brasil, no período de 2006 a 2010. Ciênc. Rural 2013;43(1):81-86.

[21] Bueno TGD, Gatti RR. Análise das principais atividades realizadas em Unidades de Alimentação e Nutrição (UAN) de serviços à la carte e self-service 2011. Guarupuava: Universidade Estadual do Centro Oeste - UNICENTRO; 2011. Trabalho de Conclusão de Curso em Nutrição. 